\title{
Impact du carbone-14 autour des centrales nucléaires EDF
}

\author{
B. LE GUEN ${ }^{1}$, F. SICLET ${ }^{2}$
}

(Manuscrit reçu le 5 novembre 2008, accepté le 18 septembre 2009)

\begin{abstract}
RÉSUMÉ Bien que le carbone-14 soit un radionucléide existant essentiellement à l'état naturel, sa présence dans l'environnement est souvent associée à la production d'électricité d'origine nucléaire. À l'occasion de la construction du nouveau réacteur EPR à Flamanville et des renouvellements réglementaires d'autorisations de rejets pour des sites existants, cette note a pour objectif de résumer la connaissance scientifique, les mesures réalisées autour des sites et les études d'impact dans l'environnement et chez l'homme pour ce radionucléide.

ABSTRACT Impact of carbon-14 due to EDF nuclear power plants release.

Although the radionuclide carbon-14 is found in their natural state, its presence in the environment is often associated with nuclear power generation. With the construction of the new EPR reactor at Flamanville underway, and the renewal of release permits for existing sites, this brief note seeks to provide a summary of scientific facts, measurements taken around nuclear sites and impact studies regarding the impact assessment of this radionuclide on man and the environment.
\end{abstract}

Keywords: Carbon14 / dosimetry / environmental impact / NPP

\section{Forme du carbone}

Le carbone-14 a deux origines, la première naturelle par action des neutrons cosmiques sur les atomes d'azote $(\approx 98 \%$ des stocks), la seconde due aux activités humaines $(\approx 2 \%)$. Ce radionucléide naturel est incorporé chaque jour par l'organisme humain.

Le carbone est un des quatre éléments (carbone, hydrogène, oxygène, azote) essentiels au développement de toute vie, il est présent, soit sous forme d'isotopes stables (carbone-12 $(\approx 99 \%)$, carbone-13 $(\approx 1 \%))$, soit sous forme d'isotope radioactif, le carbone-14 $\left({ }^{14} \mathrm{C}\right)$. Celui-ci présente la particularité de n'émettre qu'un seul type de rayonnement (bêta de faible énergie, maximum de $156 \mathrm{keV}$ ).

Dans la nature, le carbone-14 existe principalement sous forme minérale, de gaz carbonique $\left(\mathrm{CO}_{2}\right)$ dans l'atmosphère et de carbonates dans l'eau, puis sous forme organique après assimilation par les plantes au cours de la photosynthèse.

1 EDF, DIN/CEIDRE, 2 rue Ampère, 93206 Saint-Denis Cedex 1, France.

2 EDF R\&D, Laboratoire National d'Hydraulique et Environnement, BP 49, 78401 Chatou Cedex, France. 
C'est grâce à l'existence de carbone-14 à l'état naturel que la technique de datation d'un échantillon biologique a pu être utilisée ; elle tient justement compte de la décroissance radioactive du ${ }^{14} \mathrm{C}$ (5 700 ans environ) et de la connaissance précise du taux de ${ }^{14} \mathrm{C}$ atmosphérique au moment où vivait le fossile à dater. Le carbone-14 est la deuxième source (3 000-3 500 Bq), après le potassium-40 en activité de radioactivité naturelle dans le corps humain mais, en masse, la quantité est très faible en comparaison avec le carbone stable (un atome de ${ }^{14} \mathrm{C}$ pour $10^{12}$ atomes de carbone stable).

\section{Origines du carbone-14}

Le stock de carbone-14 à l'échelle de la planète est à ce jour de 12700 PBq $\left(1 \mathrm{PBq}=10^{15} \mathrm{~Bq}\right)$. Cette activité est répartie de la façon suivante : $90 \%$ dans les profondeurs des océans, $1 \%$ dans l'atmosphère et $9 \%$ à la surface des mers, dans les sédiments et la biosphère (UNSCEAR, 1996).

\subsection{Le carbone-14 d'origine humaine}

Il est dû essentiellement aux essais nucléaires atmosphériques pratiquées entre 1945 et $1980\left(\approx 200 \mathrm{PBq}\left(1 \mathrm{PBq}=10^{15} \mathrm{~Bq}\right)\right)$. Le ${ }^{14} \mathrm{C}$ rejeté par les installations nucléaires ne représente qu'environ $3 \mathrm{PBq}$ depuis l'origine du développement nucléaire (UNSCEAR, 2000).

L'estimation des rejets actuels de l'industrie nucléaire est de $168 \mathrm{TBq} / \mathrm{an}$ ( $\left.1 \mathrm{TBq}=10^{12} \mathrm{~Bq}\right)$ d'après l'UNSCEAR (2000) soit :

- $112 \mathrm{TBq} / \mathrm{an}$ par les centrales nucléaires,

- et $56 \mathrm{TBq} / \mathrm{an}$ par les usines de retraitement dont La Hague (27 TBq/an) (OSPAR, 2006) et Sellafield (17 TBq/an) (OSPAR, 2005).

L'estimation de l'AIEA (IAEA, 2004) pour les émissions des centrales nucléaires (289 TBq/an) est beaucoup plus élevée que celle de l'UNSCEAR, notamment en raison de différences dans l'estimation des rejets des filières CANDU (réacteurs à eau lourde) et VVER (réacteur à eau sous pression de technologie russe).

\subsection{Les rejets des centrales nucléaires françaises}

Dans les centrales nucléaires équipées de réacteur à eau sous pression, le carbone14 est essentiellement formé par activation neutronique de l'oxygène-17 et de l'azote-14 contenus dans l'eau du circuit primaire et dans le combustible, la quantité produite dépendant directement de l'énergie produite par le réacteur. 
TABLEAU I

Estimations des rejets de carbone-14 dans l'environnement en France (en TBq/an). Examples of carbon-14 releases to the environment in France (in TBq/year).

\begin{tabular}{ccc}
\hline $\begin{array}{c}\text { Source de carbone-14 } \\
\text { Centrales nucléaires }\end{array}$ & $\begin{array}{c}\text { Liquide en } 10^{12} \mathrm{~Bq} / \mathrm{an}(\mathrm{TBq} / \mathrm{an}) \\
\text { par réacteur }\end{array}$ & $\begin{array}{c}\text { Gazeux en } 10^{12} \mathrm{~Bq} / \mathrm{an}(\mathrm{TBq} / \mathrm{an}) \\
\text { par réacteur }\end{array}$ \\
\hline réacteur $900 \mathrm{MWe}$ & 0,011 & 0,15 \\
réacteur $1300 \mathrm{MWe}$ & 0,016 & 0,22 \\
\hline
\end{tabular}

Seul le carbone-14 formé dans l'eau de refroidissement du cœur est à l'origine des rejets des centrales. Le carbone-14 produit dans le combustible reste confiné dans les crayons et n'est rejeté sous forme gazeuse et liquide que lors des opérations de retraitement du combustible usé et celui formé dans les éléments de structure reste dans l'installation jusqu'à la déconstruction.

Les rejets gazeux de carbone-14 des centrales rapportés à l'énergie produite varient selon les types de réacteurs : d'après l'UNSCEAR (2000), ils sont de $220 \mathrm{GBq} / \mathrm{GWe}$.an $\left(1 \mathrm{GBq}=10^{9} \mathrm{~Bq}\right)$ pour les réacteurs à eau pressurisée, 510 GBq/GWe.an dans les réacteurs à eau bouillante, 1400 GBq/GWe.an dans les réacteurs graphite-gaz et $1600 \mathrm{GBq} / \mathrm{GWe}$.an pour les réacteurs à eau lourde.

Pour les REP français, les rejets gazeux sont estimés à $200 \mathrm{GBq} / \mathrm{GWe}$.an $\left(1 \mathrm{GBq}=10^{9} \mathrm{~Bq}\right)$, et les rejets liquides à $15 \mathrm{GBq} / \mathrm{GWe}$.an soit pour l'ensemble des sites électronucléaires EDF $10 \mathrm{TBq} / \mathrm{an}\left(1 \mathrm{TBq}=10^{12} \mathrm{~Bq}\right)$ (soit 60 grammes) de carbone-14 par voie atmosphérique et, et $0,7 \mathrm{TBq} / \mathrm{an}$ (soit 4 grammes) de carbone14 par an par voie liquide.

Dans les REP, le carbone-14 rejeté par voie atmosphérique se trouve :

- soit sous forme hydrogénée (méthane, éthane),

- $\quad$ soit sous forme oxydée (gaz carbonique).

La répartition est estimée à $20 \%$ sous forme de gaz carbonique et $80 \%$ sous forme organique (EPRI, 1995).

Dans les centrales françaises, la mesure systématique du carbone-14 dans les rejets a été introduite progressivement, depuis 1999, dans le cadre des renouvellements d'arrêtés réglementant les rejets. Cette évolution de la réglementation reflète les modifications de la composition des rejets. En effet, étant donné les efforts des industriels pour réduire les rejets des autres radionucléides, les rejets de carbone-14 sont devenus prépondérants dans la dose (très faible) reçue par le public autour des centrales nucléaires.

La mesure du carbone-14 dans les effluents gazeux a nécessité de compléter les dispositifs d'échantillonnage en continu à la cheminée par un système de prélèvement sur tamis moléculaire (structure zéolite). Le tamis moléculaire ne 
fixant que le carbone sous forme $\mathrm{CO}_{2}$, l'effluent est oxydé dans un four avec catalyseur en amont du tamis pour récupérer toutes les formes chimiques de carbone-14. La cartouche de tamis moléculaire est extraite trimestriellement de la chaîne de prélèvement pour être traitée en laboratoire. Le carbone-14 est désorbé de la cartouche à haute température puis précipité sous forme de carbonates et soumis à la mesure par scintillation liquide (Samson et Siclet, 2002).

La mesure du carbone-14 dans les effluents liquides est réalisée sur les échantillons prélevés dans les cuves d'effluents radioactifs avant rejet. La méthode retenue est basée sur l'utilisation d'un système de combustion de type « oxidizer». Le gaz carbonique dégagé après combustion est piégé dans une colonne remplie d'un absorbant spécifique. La colonne est ensuite rincée par le liquide scintillant qui est soumis au comptage par scintillation liquide (Samson et Siclet, 2002).

\subsection{Le carbone-14 dans l'environnement}

Dans le milieu naturel, le carbone-14 est présent dans tous les êtres vivants avec la même activité spécifique (Bq par unité de masse de carbone) que celle du milieu dans lequel ils vivent. Cette activité relativement constante au cours des derniers millénaires a subi de grandes fluctuations depuis le début de l'ère industrielle. En effet, les rejets de $\mathrm{CO}_{2}$ lié à l'utilisation de combustibles fossiles, dépourvus de ${ }^{14} \mathrm{C}$, en constante augmentation, ont diminué l'activité spécifique du ${ }^{14} \mathrm{C}$ présent dans l'atmosphère (effet de dilution isotopique généralement connu sous le nom de l'effet Suess). À l'inverse les rejets de ${ }^{14} \mathrm{C}$ d'origine humaine ont augmenté l'activité atmosphérique. L'activité en ${ }^{14} \mathrm{C}$ dans l'atmosphère est ainsi passée de $226 \mathrm{~Bq}$ carbone-14/kg de carbone au milieu du $19^{\mathrm{e}}$ siècle à un maximum de $400 \mathrm{~Bq}$ de carbone-14/kg de carbone dans les années soixante suite aux tirs nucléaires atmosphériques. Le niveau atmosphérique actuel d'environ $240 \mathrm{~Bq}$ de carbone-14/kg de carbone continue à décroître en raison de la dilution par les émissions de carbone fossile.

Dans l'environnement des centrales (JORF, 1995), les exploitants procèdent, en complément aux mesures dans les effluents à des mesures de contrôle réglementaire dans l'environnement (de l'ordre de 20000 contrôles par an et par site : cela va de mesures dans l'eau en aval des rejets, aux mesures dans le lait de vache, dans l'herbe des prés avoisinants, ...). Des contrôles sont également faits par l'IRSN, d'autre part les contrôles sont vérifiés régulièrement par l'ASN, notamment à l'occasion des visites de surveillance que fait l' $\mathrm{ASN}^{3}$ sur les sites

\footnotetext{
3 L'Autorité de sûreté nucléaire réalise régulièrement sur les sites de production EDF des visites d'une journée, le plus souvent à thème, sur la sûreté, la radioprotection, l'environnement, ... À la suite de ces visites, l'ASN réalise une lettre de suite vers l'exploitant, et ces lettres sont consultables sur son site Internet.
} 
EDF. Pour le carbone-14, le contrôle trimestriel porte sur des prélèvements de végétaux, ce bioindicateur s'étant montré plus fiable et plus simple à mettre en œuvre que la méthode de prélèvement d'air avec des barboteurs (Gontier et al., 2002). La mesure sur les végétaux est effectuée par scintillation liquide après combustion en «oxydizer». La concentration attendue hors influence des rejets est d'environ $120 \mathrm{~Bq} / \mathrm{kg}$ de végétal sec, correspondant à une activité spécifique de $240 \mathrm{~Bq} / \mathrm{kg}$ de carbone.

La principale source d'information sur les niveaux en carbone-14 dans l'environnement des centrales françaises provient des bilans radioécologiques décennaux et du programme de recherche EDF-IRSN dans des zones ateliers terrestres et aquatiques (Fournier et al., 2002; Gontier et al., 2002; Masson, 2006). Ces études qui ont démarré en 1994 font appel à des méthodes d'analyse performantes (synthèse benzénique suivie de scintillation liquide, ou synthèse de graphite suivie de spectrométrie de masse accélérée) qui permettent d'atteindre une précision de $1 \%$. L'exploitation de l'ensemble des résultats disponibles (230 échantillons dans le domaine terrestre), montre la faible influence des rejets atmosphériques de carbone-14. En effet, les mesures effectuées sur les végétaux dans les zones influencées par les rejets révèlent une augmentation en moyenne de $3 \%$ par rapport aux zones non influencées (Roussel-Debet et al., 2006).

En revanche, le marquage du milieu aquatique est plus important :

- dans les cours d'eau (180 échantillons),

les concentrations dans les poissons peuvent atteindre $800 \mathrm{~Bq}$ de carbone$14 / \mathrm{kg}$ de carbone, à l'aval proche des rejets des centrales, soit 3 à 4 fois les niveaux naturels. En moyenne, l'élévation de concentration entre l'amont et l'aval d'un site électronucléaire est de $150 \mathrm{~Bq} / \mathrm{kg}$ de carbone,

- le long des côtes de la Manche (160 échantillons),

les rejets de carbone-14 de l'industrie nucléaire se traduisent par une augmentation moyenne de l'activité en ${ }^{14} \mathrm{C}$ des poissons, mollusques et crustacés d'environ $100 \mathrm{~Bq} / \mathrm{kg}$ de carbone, principalement liée à l'usine de retraitement de La Hague.

\subsection{L'évaluation de l'exposition du public}

D'après l'UNSCEAR (2000), la moyenne mondiale de la dose efficace reçue par an et par individu résultant de la dispersion atmosphérique du carbone-14 des essais aériens est passée de 7,7 $\mu \mathrm{Sv}$ en 1963 à $1,7 \mu \mathrm{Sv}$ en 2000. Les doses correspondant aux rejets industriels sont estimées entre 0,1 et $0,2 \mu \mathrm{Sv}$ /an.

À proximité des centrales françaises, l'impact des rejets radioactifs d'une centrale nucléaire (auquel le carbone-14 peut contribuer à hauteur de $80 \%$ ), est de l'ordre du microsievert en moyenne pour les personnes les plus exposées 
TABLEAU II

Production annuelle de carbone-14 dans le monde (UNSCEAR, 2000). Annual global production of carbon-14.

\begin{tabular}{lc}
\hline Production annuelle de carbone-14 & En 10 ${ }^{12} \mathrm{~Bq} / \mathrm{an}$ (TBq/an) \\
\hline d'origine naturelle (variable liée à l'activité solaire) & 1500 \\
d'origine nucléaire (rejets atmosphérique et liquide) & 170 \\
d'origine médicale (rejet atmosphérique) & 50 \\
\hline
\end{tabular}

(c'est-à-dire habitant toute l'année au plus près des clôtures de la centrale et consommant les produits locaux : productions agricoles, eau, poissons pêchés à proximité des ouvrages de rejet) si l'on se base sur les calculs d'impact à partir des rejets réels, soit 12 fois plus faible que la dose due au ${ }^{14} \mathrm{C}$ d'origine naturelle.

La modélisation utilisée pour calculer l'exposition du public tient compte de la forme physico-chimique du carbone-14 rejeté par les centrales, qui peut être soit sous forme oxydée $\left({ }^{14} \mathrm{CO}_{2}\right)$, soit sous forme réduite. Sur la base des données internationales (EPRI, 1995), la répartition du carbone-14 dans les rejets gazeux des REP est estimée à $80 \%$ sous forme réduite $\left({ }^{14} \mathrm{CH}_{4}\right.$ notamment) et $20 \%$ sous forme de ${ }^{14} \mathrm{CO}_{2}$. Seul le carbone-14 sous forme de ${ }^{14} \mathrm{CO}_{2}$, est incorporé à la matière organique des végétaux au cours de la photosynthèse. En revanche, les deux formes interviennent dans l'exposition de l'homme par inhalation, avec pour la forme $\mathrm{CO}_{2}$, un facteur de dose deux fois plus élevé que pour les formes organiques $\left(6,2 \times 10^{-12} \mathrm{~Sv}\right.$ par Bq pour le gaz carbonique contre $2,9 \times 10^{-12} \mathrm{~Sv}$ par $\mathrm{Bq}$ pour les formes organiques). Dans les rejets liquides, en l'absence de données quantitatives sur les différentes formes de ${ }^{14} \mathrm{C}$, on considère que tout le ${ }^{14} \mathrm{C}$ est assimilable par photosynthèse dans le phytoplancton. À l'équilibre, l'activité spécifique en ${ }^{14} \mathrm{C}$ (Bq par unité de masse de carbone) dans le phytoplancton est égale à l'activité spécifique de l'eau; cette activité spécifique est ensuite conservée dans tous les maillons de la chaîne trophique (mollusques, crustacés, poissons) (IAEA, 2009). Dans les végétaux terrestres, l'activité spécifique (Bq par unité de masse de carbone) est égale à celle du ${ }^{14} \mathrm{C}$ sous forme de $\mathrm{CO}_{2}$ dans l'air ((Bq de ${ }^{14} \mathrm{C}$ sous forme $\mathrm{CO}_{2} / \mathrm{m}^{3}$ d'air)/(masse de carbone sous forme de $\mathrm{CO}_{2} / \mathrm{m}^{3}$ d'air $\left.{ }^{4}\right)$.

Dans les études de sensibilité nécessairement enveloppe réalisées dans le cadre des études d'impact des rejets radioactifs des centrales EDF, l'hypothèse de $100 \%$ $\mathrm{du}{ }^{14} \mathrm{C}$ sous forme de $\mathrm{CO}_{2}$ conduit à surestimer la dose due aux rejets gazeux qui

\footnotetext{
4 La concentration en $\mathrm{CO}_{2}$ dans l'air est souvent exprimée en ppmv, c'est-à-dire en volume de $\mathrm{CO}_{2}$ par unité volume d'air $\times 10^{6}$. On en déduit que $1 \mathrm{ppmv}=5,2 \times 10^{-4} \mathrm{~g}$ de $\mathrm{C}$ par $\mathrm{m}^{3}$. En 2005, la concentration moyenne en $\mathrm{CO}_{2}$ était de 380 ppmv soit $0,20 \mathrm{~g}$ de $\mathrm{C}$ par $\mathrm{m}^{3}$.
} 
TABLEAU III

Données biocinétiques pour le carbone organique et le monoxyde de carbone d'après la publication 67 de la CIPR (ICRP, 1993) valables pour le public.

Biocinetic data for organic carbon and monoxide carbon according to ICRP publication 67 (1993) valid for the public.

\begin{tabular}{ccc}
\hline Âge & $\begin{array}{c}\text { Période biologique (jours) } \\
\text { du carbone organique }\end{array}$ & $\begin{array}{c}\text { Période biologique (jours) } \\
\text { du monoxyde de carbone }\end{array}$ \\
\hline 3 mois & 8 & 0,14 \\
1 an & 15 & 0,14 \\
5 ans & 19 & 0,14 \\
10 ans & 26 & 0,14 \\
15 ans & 32 & 0,14 \\
\hline Adulte & 40 & 0,14 \\
\hline
\end{tabular}

atteint alors quelques microsieverts pour les personnes les plus exposées. Néanmoins, cette valeur reste faible, on peut la comparer au seuil de 10-20 microsieverts défini par l'AIEA au-dessous duquel le risque - si tant est qu'il existe pour ces faibles valeurs - est considéré comme négligeable (au sens anglo-saxon du terme : not important, not serious, not worth considering) et est très au-dessous de l'exposition naturelle de la population française (2 400 microsieverts).

\section{Aspects biologiques et sanitaires}

\subsection{En radiotoxicologie}

Chez l'homme la source de carbone est alimentaire. On estime que les apports annuels naturels en carbone-14 chez l'homme sont de $22000 \mathrm{~Bq}$, soit 0,13 $\mu \mathrm{g} / \mathrm{an}$. Quantité à comparer aux $300 \mathrm{~g}$ de carbone sous forme organique d'un apport alimentaire moyen chez l'adulte. La dose annuelle d'origine naturelle a été estimée à $12 \mu \mathrm{Sv}$. C'est le deuxième contributeur à la dose naturelle du corps humain après le potassium- 40 .

\subsubsection{Modèle biocinétique}

Le modèle biocinétique pour le carbone suppose que tous les composés organiques marqués sont distribués rapidement et uniformément dans tous les organes et tissus du corps et sont retenus avec une période biologique de 40 jours. Ces données, reprises dans la publication 67 de la CIPR (ICRP, 1993), ont été utilisées pour le calcul des coefficients de dose par inhalation et ingestion dans la publication 68 de la CIPR (ICRP, 1994) pour le travailleur, et dans les publications 72 de la CIPR (ICRP, 1996) pour les membres du public (adultes, enfants et nourrissons). 
Il n'y a pas d'informations spécifiques sur l'excrétion du carbone. On considère que l'excrétion du carbone n'entraînera pas de dose supplémentaire significative à la vessie, au côlon et à l'intestin grêle et on suppose donc qu'ils recevront des doses identiques à celles des autres tissus.

Chez l'homme, le carbone-14 sous forme de carbone organique a le même comportement biologique que le carbone stable qui est renouvelé selon une période biologique variable allant de 8 à 40 jours chez l'adulte. L'élimination du carbone se fait essentiellement sous forme de $\mathrm{CO}_{2}$ exhalé ( $270 \mathrm{~g}$ ), en comparaison les quantités contenues dans les urines et les selles étant très faibles.

\subsubsection{Monoxyde de carbone}

Le monoxyde de carbone, de formule $\mathrm{CO}$, gazeux dans les conditions ordinaires et inodore, est classé comme vapeur soluble de type SR-1. L'absorption systémique est instantanée.

\subsubsection{Dioxyde de carbone}

Le dioxyde de carbone, de formule $\mathrm{CO}_{2}$, est classé comme vapeur de type SR-2. On considère qu'il est complètement absorbé par les poumons.

\subsubsection{Méthane}

Le méthane, de formule $\mathrm{CH}_{4}$, est classé comme vapeur de type SR-2. On considère que seulement $1 \%$ du méthane inhalé est rapidement absorbé par les poumons et que la moitié est transformée en dioxyde de carbone et l'autre moitié en carbone organique.

\subsection{En radiobiologie}

La transformation spontanée du carbone-14 en azote-14 (azote stable), produit l'émission d'un rayonnement bêta qui peut provoquer des cassures des molécules en particulier de l'ADN. Le radiocarbone est d'énergie plus importante (157 keV) que le tritium $(20 \mathrm{keV})$ et sa portée dans un milieu tissulaire a été estimée à $33 \mu \mathrm{m}$ (parcours maximal dans l'air : $200 \mu \mathrm{m}$ ).

Son schéma de désintégration est : ${ }^{14} \mathrm{C} \rightarrow{ }^{14} \mathrm{~N}+\mathrm{e}^{-}+$antineutrino (Delacroix et al., 2004).

Quelques études (LeMotte et Little, 1984) utilisant directement des précurseurs de l'ADN marqués au carbone-14 au contact de cellules en division, ont mis en évidence de rares cassures de l'ADN. Cependant il a été montré que les cassures 
d'ADN observés avec le carbone-14 ont une fréquence plus faible que celles observées, à dose égale, avec d'autres types de rayonnements (comme le rayonnement gamma) ou d'autres radionucléides émetteurs bêta comme le tritium (Saintigny et al., 2008).

Il semble par ailleurs que contrairement à une irradiation externe à haut débit de dose, en cas d'incorporation de tritium ou de carbone-14 on observe peu de dommages simultanés dans une cellule et la cellule reste capable de les gérer. Cependant, ces dommages peuvent provoquer une instabilité génétique liée à un trop grand nombre d'événement de recombinaison (Saintigny et al., 2008). Hormis ces situations, qui ne correspondent pas en exposition à des expositions environnementales réalistes, il n'y a pas à ce jour d'effet observé chez l'homme dû à une exposition au ${ }^{14} \mathrm{C}$.

En particulier, Il n'est pas observé d'effet biologique après une exposition par inhalation de $\mathrm{CO}_{2}$ marqué au ${ }^{14} \mathrm{C}$ ou par ingestion de molécules organiques marquées au ${ }^{14} \mathrm{C}$.

Les études expérimentales, même après exposition à des concentrations élevées de carbone-14, ne mettent pas non plus en évidence d'effet sur la santé. Il n'est pas non plus recensé d'exposition professionnelle ou accidentelle conduisant à l'incorporation de grandes quantités de carbone-14.

\section{Conclusion}

Le carbone-14 est avant tout une radionucléide produit naturellement, les centrales nucléaires à eau sous pression produisant du carbone-14 par l'intermédiaire de plusieurs réactions d'activation. La production artificielle de carbone-14 liée aux centrales nucléaires dans le monde est actuellement équivalente à $15 \%$ de la production naturelle. Ces rejets d'effluents liquides et gazeux de ${ }^{14} \mathrm{C}$ représentent environ $75 \%$ de la quantité totale de la radioactivité rejetée mais le calcul d'impact potentiel pour les populations les plus exposées est inférieur à $1 \mu \mathrm{Sv} / \mathrm{an}$ et 12 fois plus faible que la dose due au ${ }^{14} \mathrm{C}$ produit naturellement.

Les recherches biologiques ont été relancées ces dernières années avec l'apport de la biologie moléculaire, et expérimentalement des cassures double brins ont été mises en évidence mais au plan sanitaire, aucun effet sur l'homme n'a jamais été observé. Néanmoins, il est important de compléter nos connaissances sur ce radionucléide et par conséquence encourager à ce que de nouveaux programmes de recherche en radiotoxicologie tant en France qu'au niveau européen étudie notamment après assimilation, l'impact à très faible dose $\mathrm{du}{ }^{14} \mathrm{C}$ sur l'homme et son environnement. 
Remarque des auteurs: cette publication a été préparée dans le cadre des activités du Conseil de radioprotection d'EDF.

\section{RÉFÉRENCES}

Delacroix D., Guerre J.-P., Leblanc P. (2004) Guide pratique Radionucléides et Radioprotection. EDP Siences, Les Ulis, ISBN : 2-86883-704-2.

EPRI (1995) TR-105715, project 2724-04, final report: Characterization of carbon-14 generated by the nuclear power industry.

Fournier M., Gontier G., Siclet F. (2002) La distribution du carbone-14 en milieu continental, Journée SFRP, 18 avril 2002, Paris.

Gontier G., Fournier M., Siclet F. (2002) Cycle biogeochimique du carbone-14 et du tritium dans l'environnement des C.N.P.E. français: zones ateliers de la Seine (Nogent) et de la Loire (Dampierre et Belleville), Rapport IRSN/DPRE/SERNAT 02-08.

IAEA (2004) TRS-421, Management of waste containing tritium and carbon-14.

IAEA (2009) Revision of Technical Report Series 364, Chapter 12: Specific activity models and parameter values for tritium, ${ }^{14} \mathrm{C}$ and ${ }^{36} \mathrm{Cl}$, Vienna AIEA (sous presse).

ICRP Publication 67 (1993) Age dependent doses to members of the public from intake of radionuclides: Part 2 Ingestion dose coefficients, Ann. ICRP 23 (3-4).

ICRP Publication 68 (1994) Dose Coefficients for Intakes of Radionuclides by Workers, Ann. ICRP 24 (4).

ICRP Publication 72 (1996) Age dependent doses to members of the public from intake of radionuclides: Part 5. Compilation of ingestion and inhalation dose coefficients, Ann. ICRP 26 (1).

JORF (1995) Décret n ${ }^{\circ}$ 95-540 du 4 mai 1995 relatif aux rejets d'effluents liquides et gazeux et aux prélèvements d'eau des installations nucléaires de base.

LeMotte P.K., Little J.B. (1984) DNA damage induced in human diploid cells by decay of incorporated radionuclides, Cancer Res. 44 (4), 1337-1342.

Masson M. (2006) Rapport final : carbone-14 et tritium le long des côtes de l'Atlantique et de la Manche, Rapport IRSN/DEI/SECRE-06-43.

OSPAR Commission (2005) UK report on implementation of PARCOM recommendation 91/4 on radioactive discharges.

OSPAR Commission (2006) Rapport national français de mise en œuvre de la recommandation PARCOM 91/4 sur les rejets radioactifs.

Roussel-Debet S., Gontier G., Fournier M., Siclet F. (2006) Distribution of carbon-14 in the terrestrial environment close to French nuclear power plants, J. Environ. Radioact. 87, 246-259.

Saintigny Y., Roche S., Meynard D., Lopez B.S. (2008) Homologous Recombination is Involved in the repair Response of Mamalian Cells to low doses of Tritium, Radiat. Res. 170, 172-183.

Samson T., Siclet F. (2002) EDF et le carbone-14, Journée SFRP, 18 avril 2002, Paris.

UNSCEAR (1996) Sources and Effects of Ionizing Radiation, United Nations Scientific Committee on the Effects of Atomic Radiation, Report to the General Assembly, with scientific annex, United Nations sales publication E.96.IX.3. United Nations, New York.

UNSCEAR (2000) Sources and Effects of Ionizing Radiation, United Nations Scientific Committee on the Effects of Atomic Radiation, Report to the General Assembly, with scientific annex. Part 1, annexC United Nations, New York. 